\title{
Recent advances in the diagnosis of malignant hyperthermia susceptibility: How confident can we be of genetic testing?
}

\author{
RL Robinson ${ }^{1}$, MJ Anetseder ${ }^{2}$, V Brancadoro ${ }^{3}, \mathrm{C}$ van Broekhoven ${ }^{4}$, A Carsana ${ }^{5}$, K Censier ${ }^{6}$, \\ G Fortunato $^{5}$, T Girard ${ }^{6}$, L Heytens ${ }^{7}$, PM Hopkins ${ }^{1}$, K Jurkat-Rott ${ }^{8}$, W Klinger ${ }^{8}$, \\ G Kozak-Ribbens ${ }^{9}$, R Krivosic ${ }^{10}$, N Monnier ${ }^{11}$, Y Nivoche ${ }^{12}$, D Olthoff ${ }^{13}$, H Rueffert ${ }^{13}$, \\ $\mathrm{V}_{\text {Sorrentino }}{ }^{14}, \mathrm{~V}^{\text {Tegazzin }}{ }^{15}$ and CR Mueller ${ }^{16}$
}

\begin{abstract}
${ }^{1}$ MH Investigation Unit, Academic Unit of Anaesthesia, St James University Hospital, Leeds, LS 97TF, UK; ${ }^{2}$ Klinik für Anaesthesiologie der Universität Würzburg, J-Schneider-St. 2, D-97080, Würzburg, Germany; ${ }^{3}$ Instituto de Anestesia e Rianimazione, Facolta de Medicina e Chirugia, Via S Pansini 5, I-80131 Napoli, Italy; ${ }^{4}$ Department of Biomedical Sciences, University Hospital Antwerp, Wilrijkstraat 10, B-2650 Edegem, Belgium; ${ }^{5}$ Dipartimento di Biochemica, Biotecnologie Mediche, Via S Pansini 5, I-80131 Napoli, Italy; ${ }^{6}$ Departments of Anaesthesia and Research, Kantonsspital, University of Basel, 4031 Basel, Switzerland; ${ }^{7}$ Department of Intensive Care, University Hospital Antwerp, Wilrijkstraat 10, B-2650 Edegem, Belgium; ${ }^{8}$ Department of Applied Physiology, University of Ulm, D-89081 Ulm, Germany; ${ }^{9}$ Faculte de Medicine, 27 bd Jean Moulin, F 13005 Marseille, France; ${ }^{10}$ Dept d' AnesthesieReanimation, Hospital Roger Salengro, Centre Hospitalier Regional CHRU, Universitaire de Lille, Bd. Du President Jean Leclerq, F-59037-Lille, France; ${ }^{11}$ Laboratoire BECP, DBMS-CEA Grenoble, 17 rue des martyrs, F-38054, Grenoble, Cedex, France; ${ }^{12}$ Dept d'Anesthesie, Hospital Robert Debre, 48 Bd. Serurier, F-75935, Paris, France; ${ }^{13}$ Department of Anaesthesiology and Intensive Care Medicine, University of Leipzig, Liebigstr. 20a, D-04103, Leipzig, Germany; ${ }^{14}$ Molecular Medicine Section, Department of Neuroscience, University of Siena, via A Moro, 53100 Siena, Italy; ${ }^{15}$ Department of Anaesthesiology and Critical Care Medicine, S Antonio General Hospital, Via Facciolati 71, I-35127 Padova, Italy; ${ }^{16}$ Department of Human Genetics, University of Wuerzburg, Biozentrum, Am Hubland, D-97074 Wuerzburg, Germany
\end{abstract}

Malignant hyperthermia (MH) is a condition that manifests in susceptible individuals only on exposure to certain anaesthetic agents. Although genetically heterogeneous, mutations in the RYR1 gene (19q13.1) are associated with the majority of reported $\mathrm{MH}$ cases. Guidelines for the genetic diagnosis for $\mathrm{MH}$ susceptibility have recently been introduced by the European MH Group (EMHG). These are designed to supplement the muscle biopsy testing procedure, the in vitro contracture test (IVCT), which has been the only means of patient screening for the last 30 years and which remains the method for definitive diagnosis in suspected probands. Discordance observed in some families between IVCT phenotype and susceptibility locus genotype could limit the confidence in genetic diagnosis. We have therefore assessed the prevalence of $15 R Y R 1$ mutations currently used in the genetic diagnosis of MH in a sample of over 500 unrelated European $\mathrm{MH}$ susceptible individuals and have recorded the frequency of $R Y R 1$ genotype/IVCT phenotype discordance. RYR1 mutations were detected in up to $\sim 30 \%$ of families investigated. Phenotype/genotype discordance in a single individual was observed in 10 out of 196 mutation-positive

\footnotetext{
*Correspondence: RL Robinson, MH Investigation Unit, Academic Unit of Anaesthesia, St James University Hospital, Leeds LS9 7TF, UK. Tel: +44 113 206 5271/ 5274; Fax: +44 113206 4140; E-mail: medrlr@stjames.leeds.ac.uk Received 13 August 2002; revised 2 January 2003; accepted 3 January 2003
} 
families. In five families a mutation-positive/IVCT-negative individual was observed, and in the other five families a mutation-negative/IVCT-positive individual was observed. These data represent the most comprehensive assessment of $R Y R 1$ mutation prevalence and genotype/phenotype correlation analysis and highlight the possible limitations of $\mathrm{MH}$ screening methods. The implications for genetic diagnosis are discussed.

European Journal of Human Genetics (2003) 11, 342-348. doi:10.1038/sj.ejhg.5200964

Keywords: malignant hyperthermia; ryanodine receptor; in vitro contracture test; European $\mathrm{MH}$ group; genetic diagnosis

\section{Introduction}

Malignant hyperthermia (MH) is a pharmacogenetic disorder of skeletal muscle calcium regulation. ${ }^{1}$ On exposure to certain anaesthetic agents (halogenated inhalational anaesthetics and depolarising muscle relaxants) susceptible patients may experience a range of symptoms, including skeletal muscle rigidity (from masseter spasms to generalised rigidity), metabolic acidosis, tachycardia and fever, as a consequence of an abnormally high release of intracellular calcium in skeletal muscle. The condition is potentially life threatening and is one of the main causes of morbidity and mortality during general anaesthesia. ${ }^{2}$

For the last 30 years the only reliable means of assessing an individual's risk from developing $\mathrm{MH}$ by avoiding exposure to triggering anaesthetic agents, has been via an invasive procedure, the in vitro muscle contracture test (IVCT). ${ }^{3,4}$ The test requires a sample of patient skeletal muscle tissue, which is exposed in vitro to incremental doses of specific testing agents and the contracture response measured. One of the three laboratory diagnoses is possible: $\mathrm{MH}$ susceptible (MHS), MH normal (MHN) or MH equivocal (MHE). Patients classified as MHS and MHE are considered clinically at risk to $\mathrm{MH}$, although the exact correlation between MHS/MHE IVCT phenotype and clinical development of an $\mathrm{MH}$ crisis is unknown. The IVCT has been standardised across Europe by the European Malignant Hyperthermia Group (EMHG) and shows a high degree of sensitivity (99.0\%) and specificity (93.6\%). ${ }^{5}$ However, it is time consuming and expensive to perform and leaves patients with a small scar.

Genetically, MH susceptibility characterised by IVCT phenotype exhibits an autosomal dominant mode of inheritance with estimated prevalence of one in $8500 .^{2}$ The condition demonstrates both locus and allelic heterogeneity ${ }^{6,7}$ with six loci so far implicated of which four have been identified only in isolated families across Europe. ${ }^{8-12}$ However, one locus on chromosome 19q13.1, the ryanodine receptor gene (RYR1), accounts for the majority of $\mathrm{MH}$ susceptible cases with $>50 \%$ of families demonstrating linkage to this region. ${ }^{13}$

A long-term goal of the EMHG has been to assess whether DNA-based diagnosis for the condition is feasible.
For several reasons, this has proven difficult as (1) the extent of genetic heterogeneity for MH susceptibility was not well established, (2) defects in the RYR1 gene, the major susceptibility locus, were not widely characterised, (3) the majority of 'mutations' identified in the gene were missense, which made it difficult to predict the true effect of such variants on the functioning of the ryanodine receptor channel and (4) genotype-phenotype correlation analysis in $\mathrm{MH}$ susceptible families revealed discrepancies between IVCT phenotypes and RYR1 genotypes, which further compounded the confusion as to the pathogenicity of $R Y R 1$ variants identified. ${ }^{14-16}$

Following 10 years of genetic research and investigation of MH-susceptible families as a European consortium, a stage has now been reached where genetic screening for the condition can be offered in a limited capacity. ${ }^{17}$ Genetic testing guidelines have been recently published by the EMHG to enable DNA diagnosis of $\mathrm{MH}$ in supplementation to the IVCT method of patient screening in MH families. In summary, genetic testing using certain mutations in the RYR1 gene demonstrated to be 'causative' of $\mathrm{MH}$ through in vitro biochemical assays, ${ }^{18,19}$ or through linkage analysis with markers flanking the RYR1 locus is now feasible. Individuals carrying a 'causative' mutation or high-risk susceptibility haplotype are considered at risk of developing $\mathrm{MH}$ independent of IVCT diagnoses.

A main concern of introducing DNA diagnosis for the condition is the apparent discordance between IVCT phenotype and RYR1 genotype in certain MH-susceptible families. Examples include patients classified as MHS by the IVCT but where the familial mutation or high-risk susceptibility haplotype is absent. In contrast, some cases classified as MHN by the IVCT have been reported to carry the familial mutation or high-risk susceptibility haplotype. Such individuals, under current testing strategies are considered at risk for developing $\mathrm{MH}$ on the basis of genetic data alone. False-negative diagnoses are more likely to have potentially fatal consequences under anaesthesia and are considered the most significant. To minimise false-negative results through genetic diagnosis and formally exclude risk from $\mathrm{MH}$, the accepted practice of the EMHG is to use the IVCT for the characterisation of 
individuals where the familial mutation, or high-risk haplotype is absent. The IVCT also remains the definitive method of diagnosis for the assessment of suspected $\mathrm{MH}$ probands.

Here, we have assessed the incidence of phenotype/ genotype discordance across European $\mathrm{MH}$ families. We have collated data on 15 RYR1 missense mutations described in the genetic testing guidelines. The data represent investigations by 11 European $\mathrm{MH}$ groups. The results give the most comprehensive assessment of the prevalence of these mutations across European populations, providing the most thorough analysis of the concordance between clinical (IVCT) phenotype and $R Y R 1$ genotype to date and substantiate the rationale of the current DNA testing guidelines.

\section{Materials and methods}

Patients were all characterised using the European IVCT protocol. Prior to testing, the electrically invoked twitch (g) of the muscle specimen is recorded as an indicator of specimen viability. An MHS IVCT phenotype is diagnosed on the basis of an at least $0.2 / \mathrm{g}$ contracture on application of $2 \%$ halothane, and in an independent test on application of $2 \mathrm{mM}$ caffeine. Individuals classified as MHE present a positive response to one of the trigger agents only. Normal individuals (MHN) do not react at the threshold dose of either trigger agent to give a sustained contracture of $0.2 \mathrm{~g}$ or above.

Genotypic data were collated from each contributing centre concerning the screening for the 15 RYR 1 mutations described in the current genetic testing guidelines. These have all been described previously. ${ }^{20-33}$

\section{Results \\ Prevalence of $R Y R 1$ mutations in Europe}

Table 1 summarises the prevalence data for the 15 mutations from the screening of unrelated IVCT MHS individuals from Belgium, Italy, France, Germany, Switzerland and the UK. Although not all families were screened for all 15 mutations, three mutations appear to be prevalent in the European population, 1021G $>$ A, $1840 \mathrm{C}>\mathrm{T}, 7300 \mathrm{G}>\mathrm{A}$. These mutations account for 14.8 , 28.6 , and $34.2 \%$ of the mutation positive cases $(n=196)$, respectively. Table 2 shows the relative contribution of data and prevalence of mutations in the participating groups. In France, Germany and Switzerland the 1840C $>$ T mutation appears most prevalent, whereas in the UK the $7300 \mathrm{G}>\mathrm{A}$ mutation accounts for the majority of mutation-positive cases. Where a uniform number of individuals were screened the mutation detection rate was $31.6 \%$ in France, $12.0 \%$ in Switzerland and $27.0 \%$ in the UK.

\section{Discordance between $R Y R 1$ genotype and IVCT phenotype}

On segregation analysis of RYR1 mutation with IVCT phenotype in the 196 mutation-positive families, $16(8.0 \%)$ demonstrated discordance between the IVCT phenotype and $R Y R 1$ genotype. In eight families the familial mutation was not detected in an IVCT MHS individual, and in the remaining eight, the familial mutation was detected in an IVCT MHN individual. Of the 15 mutations three $(1021 \mathrm{G}>\mathrm{A}, 1840 \mathrm{C}>\mathrm{T}, 7300 \mathrm{G}>\mathrm{A})$ demonstrated phenotype/genotype discordance. These were the most prevalent mutations in the sample.

The IVCT data were re-examined in the 16 families showing discordant results. Criteria used for re-evaluation of the data in all family members included: (a) assessment

Table 1 Prevalence of $R Y R 1$ mutations and frequency of $R Y R 1$ genotype-IVCT phenotype discordance in Europe

\begin{tabular}{|c|c|c|c|c|c|c|c|}
\hline RYR1 Exon & $\begin{array}{l}\text { Mutation } \\
\text { nucleotide }\end{array}$ & Codon & $\begin{array}{l}\text { Total no. } \\
\text { of families }\end{array}$ & Prevalence & $\begin{array}{l}\text { No of families } \\
\text { discordant }\end{array}$ & $\begin{array}{l}\text { No. families with an } \\
\text { MHS individual } \\
\text { without a mutation }\end{array}$ & $\begin{array}{l}\text { No. families with } \\
\text { an MHN individual } \\
\text { with a mutation }\end{array}$ \\
\hline 6 & $487 \mathrm{C}>\mathrm{T}$ & Arg163Cys & 673 & $10(1.5 \%)$ & & & \\
\hline 9 & $742 \mathrm{G}>\mathrm{A}$ & Gly248Arg & 587 & $1(0.2 \%)$ & & & \\
\hline 11 & $1021 \mathrm{G}>\mathrm{A}$ & Gly341Arg & 737 & $29(3.9 \%)$ & 4 & 3 & 1 \\
\hline 12 & $1209 C>G$ & Ile403Met & 594 & 0 & & & \\
\hline 17 & $1840 C>T$ & Arg614Cys & 806 & $56(6.9 \%)$ & 7 & 4 & 3 \\
\hline 17 & $1841 \mathrm{G}>\mathrm{T}$ & Arg614Leu & 607 & $4(0.4 \%)$ & & & \\
\hline 39 & $6487 C>T$ & Arg2163Cys & 644 & $2(0.3 \%)$ & & & \\
\hline 39 & $6488 \mathrm{G}>\mathrm{A}$ & Arg $2163 \mathrm{His}$ & 610 & $4(0.7 \%)$ & & & \\
\hline 45 & $7300 \mathrm{G}>\mathrm{A}$ & Gly2434Arg & 717 & $67(9.3 \%)$ & 5 & 1 & 4 \\
\hline 45 & $7304 G>A$ & Arg $2435 \mathrm{His}$ & 669 & $14(2.1 \%)$ & & & \\
\hline 46 & $7372 \mathrm{C}>\mathrm{T}$ & Arg2458Cys & 610 & $5(0.8 \%)$ & & & \\
\hline
\end{tabular}


Table 2 Prevalence of $R Y R 1$ mutations across European populations

\begin{tabular}{|c|c|c|c|c|c|c|c|c|c|c|c|c|}
\hline \multirow[b]{2}{*}{ Mutation nucleotide } & \multicolumn{2}{|c|}{ Belgium } & \multicolumn{2}{|l|}{ Italy } & \multicolumn{2}{|l|}{ France } & \multicolumn{2}{|c|}{ Germany } & \multicolumn{2}{|c|}{ Switzerland } & \multicolumn{2}{|l|}{ UK } \\
\hline & No. F & Prev. & No. $F$ & Prev. & No. F & Prev. & No. F & Prev. & No. F & Prev. & No. F & Prev. \\
\hline $103 T>C$ & NT & NT & NT & NT & 106 & $1(0.9 \%)$ & 100 & 0 & 66 & 0 & 297 & 0 \\
\hline $487 \mathrm{C}>\mathrm{T}$ & 17 & 0 & 48 & $2(4 \%)$ & 106 & $2(1.9 \%)$ & 139 & $2(1.4 \%)$ & 66 & 0 & 297 & $4(1.3 \%)$ \\
\hline $742 \mathrm{G}>\mathrm{A}$ & 15 & 0 & 3 & 0 & 106 & & 100 & 0 & 66 & 0 & 297 & $1(0.3 \%)$ \\
\hline $1021 G>A$ & 24 & $4(16 \%)$ & 42 & 0 & 106 & $9(8.5 \%)$ & 202 & $3(1.4 \%)$ & 66 & 1 (1.5\%) & 297 & $12(3 \%)$ \\
\hline $1209 C>C$ & 17 & 0 & 8 & 0 & 106 & & 100 & & 66 & 0 & 297 & 0 \\
\hline $1565 A>C$ & 3 & 0 & 2 & 0 & 106 & $1(0.9 \%)$ & 100 & & 66 & 0 & 297 & 0 \\
\hline $1654 C>T$ & NT & NT & NT & NT & 106 & & 100 & & 66 & 0 & 297 & 0 \\
\hline $1840 \mathrm{C}>\mathrm{T}$ & NT & NT & 50 & $4(8 \%)$ & 106 & $12(11 \%)$ & 287 & $32(11 \%)$ & 66 & $3(4.5 \%)$ & 297 & $4(1.3 \%)$ \\
\hline $1841 \mathrm{G}>\mathrm{T}$ & NT & NT & 38 & 0 & 106 & $3(2.8 \%)$ & 100 & $1(1 \%)$ & 66 & 0 & 297 & 0 \\
\hline $6487 C>T$ & 11 & 1 (9\%) & 42 & 0 & 106 & & 100 & $1(1 \%)$ & 66 & 0 & 297 & $1(0.3 \%)$ \\
\hline $6488 \mathrm{G}>\mathrm{A}$ & NT & NT & 42 & 0 & 106 & & 100 & & 66 & 0 & 297 & $4(1.3 \%)$ \\
\hline $7300 G>A$ & NT & NT & 41 & $1(2.4 \%)$ & 106 & $3(2.8 \%)$ & 207 & $7(3.4 \%)$ & 66 & $2(3 \%)$ & 297 & $53(17.5 \%)$ \\
\hline $7304 \mathrm{G}>\mathrm{A}$ & 17 & 0 & 44 & $2(4.5 \%)$ & 106 & $1(0.9 \%)$ & 139 & $8(5.8 \%)$ & 66 & 0 & 297 & $5(2 \%)$ \\
\hline $7372 C>T$ & NT & NT & 41 & $3(7.3 \%)$ & 106 & & 139 & $2(1.4 \%)$ & 66 & $2(3 \%)$ & 297 & 0 \\
\hline $7373 G>A$ & NT & NT & 41 & 0 & 106 & $2(1.9 \%)$ & 100 & $1(1 \%)$ & 66 & 0 & 297 & 0 \\
\hline
\end{tabular}

No. F=number of families, Prev.=prevalence.

of the viability of the muscle specimen prior to testing, with good viability indicated by a pre-test twitch of $1 \mathrm{~g}$ or above, (b) reassessment of all IVCT trace measurements and (c) whether the methodology used for the IVCT conformed to the EMHG IVCT guidelines. In all discordant cases genetic data were repeated on an independent DNA sample where possible, to exclude the possibility of sample mix-up. In 10/16 families, discordance could not be explained by possible erroneous clinical or genetic data. In all 10 families, discordant phenotype/genotype data was observed in one individual. In five of these families an IVCT MHS individual did not carry the familial mutation and in five families a mutation was detected in an IVCT MHN individual. For these 10 families, of 48 IVCT-and DNA-tested individuals, 5/48 (10.4\%) were discordant MHS (dMHS) and 5/48 (10.4\%) were discordant MHN (dMHN) cases. By mutation type, of the total number of IVCT-tested individuals, $1021 \mathrm{G}>\mathrm{A}$ demonstrated $2 / 23 \mathrm{dMHS}$, and $1 / 23$ $\mathrm{dMHN} ; 1840 \mathrm{C}>\mathrm{T}$ demonstrated $2 / 20 \mathrm{dMHS}$ and $2 / 20$ $\mathrm{dMHN}$, and $7300 \mathrm{G}>\mathrm{A}$ demonstrated 1/8 dMHS and 2/8 dMHN individuals (Table 3 ). MHE classified individuals are also considered clinically at risk to $\mathrm{MH}$ and may be equated with overall weaker contractures in the IVCT, or high responders to one specific testing agent used in the test. Discordance was only observed for mutation $1021 \mathrm{G}>\mathrm{A}$, where two MHE cases did not carry the familial mutation.

In the remaining six families, it was possible that the observed discordance was attributable to (a) clinical data that did not conform to current IVCT testing criteria having been recorded prior to European standardisation of the method (LMH03), (b) poor muscle specimen viability, indicated by a low pretest twitch response prior to the IVCT, resulting in potentially false-negative IVCT diagnoses (LMH52), c) DNA sample mix-up (LMH54) or (d) border-line MHS/ MHN IVCT contracture measurements (LZB, ULM31). In one family, no clinical data were available on the discordant individual (PAMH3) (Table 3).

\section{Discussion}

In the largest retrospective study of $\mathrm{MH}$ clinical and genetic data reported, we have shown that the relative prevalence of $R Y R 1$ mutations varies in IVCT MHS patients across Europe inferring different founder events. The overall mutation detection rate between participating centres screening a uniform sample for all 15 mutations suggest that further mutations in $R Y R 1$, or mutations at other loci remain to be characterised. The data indicate that DNA testing for $\mathrm{MH}$ susceptibility in its present capacity will benefit up to $30 \%$ of $\mathrm{MH}$ families, depending on the population investigated, and provide the rationale for the development of mutation testing strategies.

The observed discordance between RYR1 genotype and IVCT phenotype is a concern from the perspective of assigning the correct diagnosis of MH status. The IVCT is conducted to achieve high sensitivity at the cost of reduced specificity, thus discordance between phenotypic and genetic data is not unexpected. The presence or absence of a mutation can be considered more definitive in the genetic diagnosis of dominant Mendelian disorders. However, current genetic diagnostic strategies for assessing $\mathrm{MH}$ status are conservative in this respect because of the fact that (1) the majority of mutations associated with $\mathrm{MH}$ susceptibility are missense, where the pathogenicity of such mutations requires careful evaluation and (2) there is evidence that additional loci/mutations may contribute to $\mathrm{MH}$ susceptibility in individual families, which may 
Table 3 European $\mathrm{MH}$ families demonstrating discordance between IVCT phenotype and RYR1 genotype

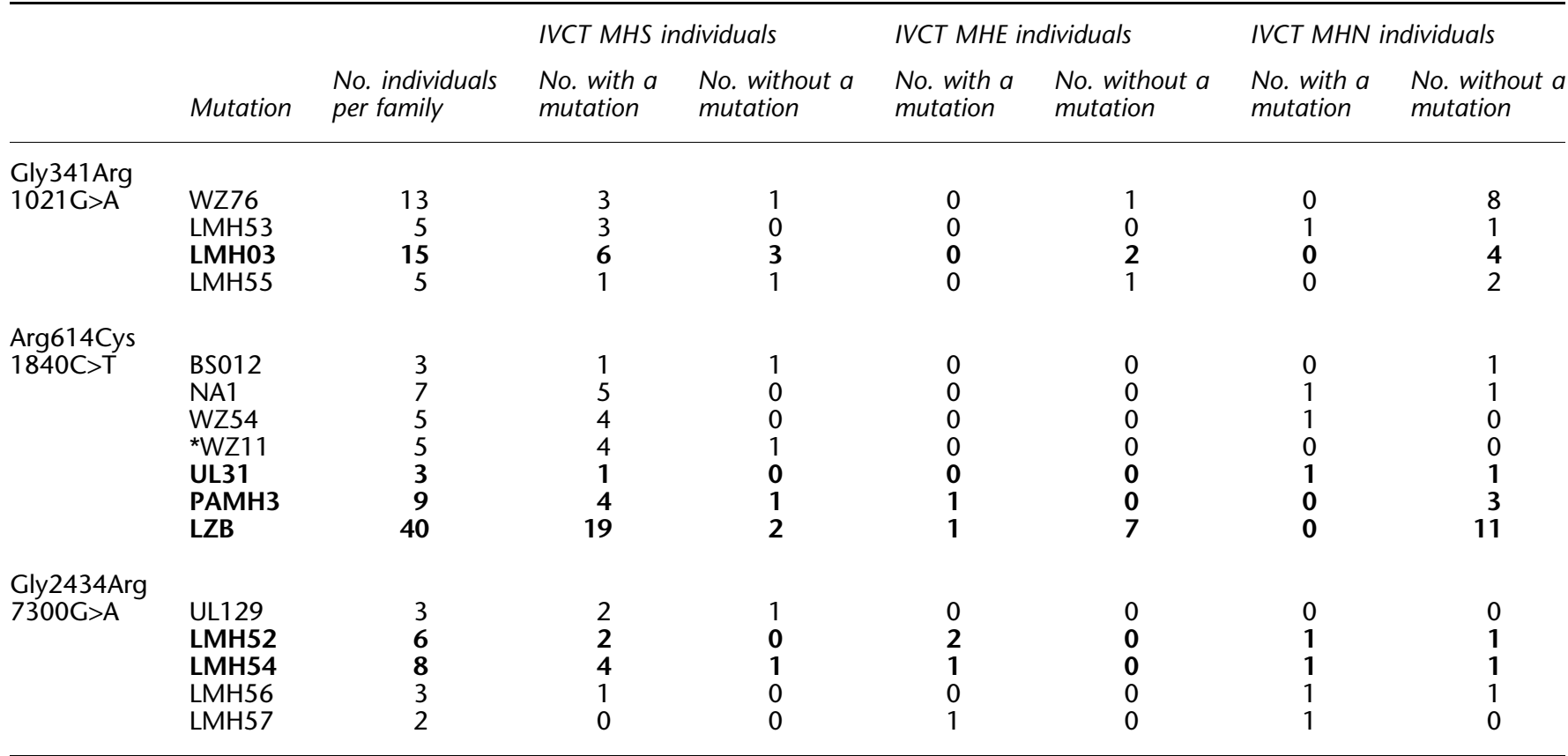

Discordant families excluded from analysis owing to: early clinical data: one family; poor twitch data: three families; DNA sample mix-up: one family; threshold contracture: one family; no IVCT (in bold) data provided: one family.

account for or modify the observed IVCT response. Therefore, genetic testing guidelines are designed to minimise the possibility of a potentially fatal false-negative $\mathrm{MH}$ diagnosis in an individual that does not carry a mutation detected in other family relatives. Considering the IVCT as the bench mark for an MH-susceptible diagnosis, the frequency of such diagnoses occurs in $2.6 \%$ $(5 / 190)$ of families or $\sim 10 \%$ of individuals tested. Conversely, $2.6 \%(5 / 190)$ of families or $\sim 10 \%$ of individuals demonstrate false-positive $\mathrm{MH}$ diagnoses, that is $\mathrm{dMHN}$, on the basis of genetic data that could reflect variability in the IVCT. The discordance rates are heavily influenced by the number of individuals in each family who have been investigated by both IVCT and DNA methods. Unfortunately, genotype/phenotype concordance data were not available on all individuals from all mutation-positive families identified $(n=196)$. It is therefore possible that the values of 10\% dMHS and 10\% dMHN (95\% CI 2-19\%) individuals are inflated. As the criteria used for the IVCT are designed to minimise false-negative $\mathrm{MH}$ diagnoses, the expectation was for the discordance rate to be greater in IVCT MHS, compared to MHN cases. However, inclusion of IVCT MHE data as a clinically at-risk group slightly elevates the frequency of discordance observed among individuals compared to the MHN group. Following the exclusion of potentially erroneous data from the analysis, and since all mutations studied have been demonstrated to confer some functional effect on the RYR1 protein through in vitro biochemical assays, mutation penetrance, genetic background, environmental effects and variability in the IVCT itself are likely to contribute to the observed discordance. ${ }^{5,34,35}$. In support of environmental effects, it has been well documented that the clinical penetrance of $\mathrm{MH}$ is varied, patients undergoing several uneventful anaesthetics before experiencing a reaction.

Only mutations $1021 \mathrm{G}>\mathrm{A}, 1840 \mathrm{C}>\mathrm{T}$ and $7300 \mathrm{G}>\mathrm{A}$ demonstrated phenotype/genotype discordance. This observation may simply reflect the relative mutation prevalence. However, it has been shown that IVCT contracture response varies with mutation genotype. Those mutations associated with weaker contractures may therefore be expected to show higher discordance rates than those associated with more severe contracture phenotypes. All three mutations have been shown to be associated with weaker contractures compared to $487 \mathrm{C}>\mathrm{T}, 6487 \mathrm{C}>\mathrm{T}$, $6488 \mathrm{G}>\mathrm{A}$ and $7304 \mathrm{G}>\mathrm{A}$ by analysis of channel mutants in vitro ${ }^{18}$ and by comparative analysis of IVCT data in mutation carriers. ${ }^{36,37}$

With over 500 individuals investigated for RYR1 mutations currently used in genetic diagnosis, this represents the most comprehensive evaluation of mutation prevalence and genotype/phenotype concordance data. We have shown that in $2.6 \%$ of families DNA tested, an individual may carry the familial RYR1 mutation, where IVCT diagnosis would be negative. This finding should be considered when patients receive counselling on receipt of DNA test results. Conversely, the fact that in 
$2.6 \%$ of families an individual may be classified 'normal' by genetic diagnosis, when in fact IVCT MHS, supports the rationale of the current DNA testing guidelines to biopsy and IVCT to all individuals found not to carry a familial mutation or high-risk susceptibility haplotype. More importantly, the data emphasise that DNA screening for $\mathrm{MH}$ susceptibility is not feasible in isolation of the IVCT because of the risk, however small, of false-negative diagnoses.

\section{References}

1 Mickelson JR, Louis CF: Malignant hyperthermiaExcitation-contraction coupling $\mathrm{Ca}^{2+}$ release channel, and cell $\mathrm{Ca}^{2+}$ regulation defects. Phys Rev 1996; 76: 537-592.

2 Hopkins PM: Malignant hyperthermia: advances in clinical management and diagnosis. Br J Anaesth 2000; 85: 118-128.

3 European Malignant Hyperpyrexia Group. A protocol for the investigation of malignant hyperpyrexia susceptibility. $\mathrm{Br} J$ Anaesth 1984; 56: 1267-1269.

4 European Malignant Hyperpyrexia Group. Laboratory diagnosis of malignant hyperpyrexia susceptibility. Br J Anaesth 1985; 57: 1038.

5 Ørding $\mathrm{H}$, Brancadoro $\mathrm{V}$, Cozzolino $\mathrm{S}$ et al: In vitro contracture test for diagnosis of malignant hyperthermia following the protocol of the European MH Group: results of testing patients surviving fulminant MH and unrelated low-risk subjects. Acta Anaesthesiol Scand 1997; 41: 955-966.

6 Fagerlund TH, Islander G, Ranklev Twetman E, Berg K: Malignant hyperthermia susceptibility, an autosomal dominant disorder? Clin Genet 1997; 51: 365-369.

7 Deufel T, Golla A, Iles D et al: Evidence for genetic heterogeneity of malignant hyperthermia susceptibility. Am J Hum Genet 1992; 50: 1151-1161.

8 Levitt RC, Olkers A, Meyes S et al: Evidence for the localisation of a malignant hyperthermia susceptibility locus (MHS2) to chromosome 17q. Genomics 1992; 14: 562-566.

9 Iles DE, Lehmann-Horn F, Scherer SW et al: Localisation of the gene encoding the $\alpha_{2} / \delta$ subunits of the L-type voltage dependent calcium channel to chromosome $7 \mathrm{q}$ and analysis of the segregation of flanking markers in malignant hyperthermia susceptible families. Hum Mol Genet 1994; 3: 969-975.

10 Sudbrak R, Procaccio V, Klausnitzer M et al: Mapping of a further malignant hyperthermia susceptibility locus to chromosome 3q13.1. Am J Hum Genet 1995; 56: 684-691.

11 Robinson RL, Monnier N, Wolz W et al: A genome wide search for susceptibility loci in three European malignant hyperthermia pedigrees. Hum Mol Genet 1997; 6: 953-961.

12 Monnier N, Procaccio V, Stieglitz P et al: Malignant hyperthermia susceptibility is associated with a mutation of the $\alpha 1$-subunit of the dihydropyridine sensitive L-type voltage dependent calcium channel receptor in skeletal muscle. Am J Hum Genet 1997; 60: 1316-1325.

13 Robinson RL, Curran JL, Hall WJ et al: Genetic heterogeneity and HOMOG analysis in British malignant hyperthermia families. $J$ Med Genet 1998; 35: 196-201.

14 Deufel T, Sudbrak R, Feist Y et al: Discordance in a malignant hyperthermia pedigree, between the in vitro contracture test phenotypes and haplotypes for the MHS1 region of chromosome $19 \mathrm{q} 12-13.2$, comprising the C1840T transition in the RYR1 gene. Am J Hum Genet 1995; 56: 1334-1342.

15 Fagerlund $\mathrm{TH}$, Ording H, Bendixen D, Islander G, Ranklev Twetman E, Berg K: Discordance between malignant hyperthermia susceptibility and RYR1 mutation C1840T in two Scandinavian MH families exhibiting this mutation. Clin Genet 1997; 52: 416-421.
16 Adeokun AM, West SP, Ellis FR et al: The G1021A substitution in the RYR1 gene does not cosegregate with malignant hyperthermia in a British pedigree. Am J Hum Genet 1997; 60: 833-841.

17 Urwyler A, Deufel T, McCarthy T, West S: Guidelines for the molecular detection of susceptibility to malignant hyperthermia. Br J Anaesth 2001; 86: 283-287.

18 Tong J, Oyamada H, Demaurex N, Grinstein S, McCarthy T, MacLennan D: Caffeine and halothane sensitivity of intracellular $\mathrm{Ca}^{2+}$ release is altered by 15 calcium release channel (ryanodine receptor) mutations associated with malignant hyperthermia and/or central core disease. J Biol Chem 1997; 272: 26332-26339.

19 Tong J, McCarthy TV, MacLennan DH: Measurement of resting cytosolic $\mathrm{Ca}^{2+}$ concentrations and $\mathrm{Ca}^{2+}$ store size in HEK-293 cells transfected with malignant hyperthermia or central core disease mutant $\mathrm{Ca}^{2+}$ release channels. J Biol Chem 1999; 274: 693-702.

20 Gillard EF, Otsu K, Fujii J et al: A substitution of cysteine for arginine 614 in the ryanodine receptor is potentially causative of human malignant hyperthermia. Genomics 1991; 11: 751-755.

21 Gillard EF, Otsu K, Fujii J et al: Polymorphisms and deduced amino acid substitutions in the coding sequence of the ryanodine receptor (RYR1) gene in individuals with malignant hyperthermia. Genomics 1992; 13: 1247-1254.

22 Keating KE, Quane KA, Manning BM et al: Detection of a novel RYR1 mutation in four malignant hyperthermia pedigrees. Hum Mol Genet 1994; 3: 1855-1858.

23 Keating KE, Giblin L, Lynch PJ et al: Detection of a novel mutation in the ryanodine receptor gene in an Irish malignant hyperthermia pedigree: correlation of the IVCT response with the affected and unaffected haplotypes. J Med Genet 1997; 34: 291-296.

24 Lynch PJ, Krivosic-Horber R, Reyford $\mathrm{H}$ et al: Identification of heterozygous and homozygous individuals with the novel $R Y R 1$ mutation Cys35Arg in a large kindred. Anesthesiology 1997; 86: 620-626.

25 Lynch PJ, Tong J, Lehane $\mathrm{M}$ et al: A mutation in the transmembrane/luminal domain of the ryanodine receptor is associated with abnormal calcium release channel function and severe central core disease. Proc Natl Acad Sci USA 1999; 96: 4164-4169.

26 Manning BM, Quane KA, Ording $\mathrm{H}$ et al: Identification of novel mutations in the ryanodine receptor gene (RYR1) in malignant hyperthermia: genotype-phenotype correlation. Am J Hum Genet 1998; 62: 599-609.

27 Phillips MS, Khanna VK, De Leon S, Frodis W, Britt BA, MacLennan DH: The substitution of Arg for Gly2433 in the human skeletal muscle ryanodine receptor is associated with malignant hyperthermia. Hum Mol Genet 1994; 3: 2181-2186.

28 Quane KA, Healy JM, Keating KE et al: Mutations in the ryanodine receptor gene in central core disease and malignant hyperthermia. Nat Genet 1993; 5: 51-55.

29 Quane KA, Keating KE, Healy JM et al: Mutation screening of the RYR1 gene in malignant hyperthermia: detection of a novel Tyr to Ser mutation in a pedigree with associated central cores. Genomics 1994; 23: 236-239.

30 Quane KA, Keating KE, Manning BM et al: Detection of a novel common mutation in the ryanodine receptor gene in malignant hyperthermia: implications for diagnosis and heterogeneity studies. Hum Mol Genet 1997; 3: 471-476.

31 Quane KA, Ording H, Keating KE et al: Detection of a novel mutation at amino acid position 614 in the ryanodine receptor in malignant hyperthermia. Br J Anaesth 1997; 79: 332-337.

32 Zhang Y, Chen HS, Khanna VK et al: A mutation in the human ryanodine receptor gene associated with central core disease. Nat Genet 1993; 5: 46-50.

33 Brandt A, Schleithoff L, Jurkat-Rott K, Klinger W, Baur C, Lehmann-Horn F: Screening of the ryanodine receptor gene in 105 malignant hyperthermia families: novel mutations and concordance with the in vitro contracture test. Hum Mol Genet 1999; 8: 2055-2062. 
34 Robinson RL, Curran JL, Ellis FR, Halsall PJ et al: Multiple interacting gene products may influence susceptibility to malignant hyperthermia. Ann Hum Genet 2000; 64: 307-320.

35 Hopkins PM, Hartung E, Wappler F: The European Malignant Hyperthermia Group: Multicentre evaluation of ryanodine contracture testing in malignant hyperthermia. Br J Anaesthesia 1991; 66: 611-613.
36 Robinson R, Brooks C, Brown SL et al: RYR1 mutations causing central core disease are associated with more severe malignant hyperthermia in vitro contracture test phenotypes. Hum Mut 2002; 20: 88-97.

37 Girard T, Urwyler A, Censier K, Mueller C, Zorzato F, Treves S: Genotype-Phenotype comparison of the Swiss malignant hyperthermia population. Hum Mut 2001; 18: 357-364. 